\title{
A $2 \times 2$ MIMO DVB-T2 System: Design, New Channel Estimation Scheme and Measurements With Polarization Diversity
}

\author{
Carlos Gómez-Calero, Luis Cuéllar Navarrete, Leandro de Haro, Member, IEEE, and \\ Ramón Martínez, Member, IEEE
}

\begin{abstract}
The increasing interest in MIMO (Multiple-Input Multiple-Output) systems has given rise to a prolific research activity in recent years. Both theoretical and practical issues have been studied. However, so far few MIMO testbeds or prototypes have been built for DVB-T or future standards. In this paper, a novel $2 \times 2$ MIMO testbed specifically designed for evaluating the performances of a DVB-T2 MIMO system is presented. The description of signal processing is detailed including a new scheme to estimate the MIMO channel matrix. Finally, measurement results with different polarization schemes are presented for typical scenarios, obtaining higher capacity in LoS situations using polarization diversity.
\end{abstract}

Index Terms-Field trials and test results, Multiple-Input Multiple-Output, signal processing for transmission.

\section{INTRODUCTION}

D $\mathrm{B}-\mathrm{T} 2$, the second generation of the DVB proposal for digital terrestrial TV, has been recently proposed by DVB project [1] as an evolution of DVB-T when the shutdown of analog television process will be finished. In order to give a newer technical response to the necessity of the digital dividend, that is the process by which some free frequencies at UHF used by analog TV will be assigned to different services (3G/4G), DVB-T2 will improve frequency efficiency to provide multicast in $\mathrm{HD}$ with the same $8 \mathrm{MHz}$ channel.

As DVB-T, DVB-T2 expects to be received in plugged TV terminals, in mobile environment or with unplugged terminals in indoor or in low speed (pedestrian) environments, so a MISO scheme has been included, transmitting with a distributed Alamouti block code. However, in order to go further a full MIMO scheme is proposed in this paper, which may be similar to the one that will be included in NGH (second generation of DVB-H) in the next future, obtaining a very efficient performance in high
Doppler environments for terminals (unplugged or not) operating in high speed vehicles.

The increasing interest in MIMO (Multiple-Input MultipleOutput) systems has given rise to a prolific research activity in this topic in recent years. Several aspects have been studied, including both theoretical and practical issues. So far much effort has been put in the study and design of coding schemes for MIMO systems, taking into account different considerations such as some knowledge (full, partial or none) of channel information at the transmitter or the type of scenario. Another point of view is the design of the antenna array and its configuration, since the MIMO systems performances depend on three aspects: antenna array, channel characteristics and coding.

The pioneer works [2], [3] show an increase in the data bit rate and an enhancement of quality of signal at the receiver. Many works can be found in the literature for the channel propagation characteristics and signal processing in MIMO [4]-[7].

On the other hand, DVB-T2 is expected to provide higher efficiencies in frequency than the nowadays DVB standard DVB-T. DVB-T2 proposal considers the inclusion of MISO technology but not MIMO. MIMO will be considered in future revisions and it could provide a further increment of frequency efficiency mainly in harsh scenarios such as strong multipath environments or highly Doppler radio channels.

Nowadays, few works employing multiple antennas at each side of the radio link for either DVB-T or DVB-T2 can be found in the literature. There are barely some research works carried out by BBC [8]-[10], as pioneer researchers using MIMO with DVB-T. In order to evaluate the performances of a DVB-T2 system in realistic scenarios, the use of a real platform is of great interest, since it enables to include several aspects that are not usually addressed in theoretical studies or simulations, such as the effect of different antennas or scenarios [11].

In this contribution, a novel $2 \times 2 \mathrm{MIMO}$ testbed for DVB-T2 has been designed and implemented in order to test the enhancements obtained by the using of multiple antennas at the transmitter and the receiver side for UHF band, particularly at frequency of $594 \mathrm{MHz}$. Moreover, MIMO measurements have been carried out by employing polarization diversity, since there are few works in the literature related to the use of polarization diversity for UHF and DVB-T [12]-[14]. Apart from that, for the future use of MIMO with DVB-T2, a new scheme for 


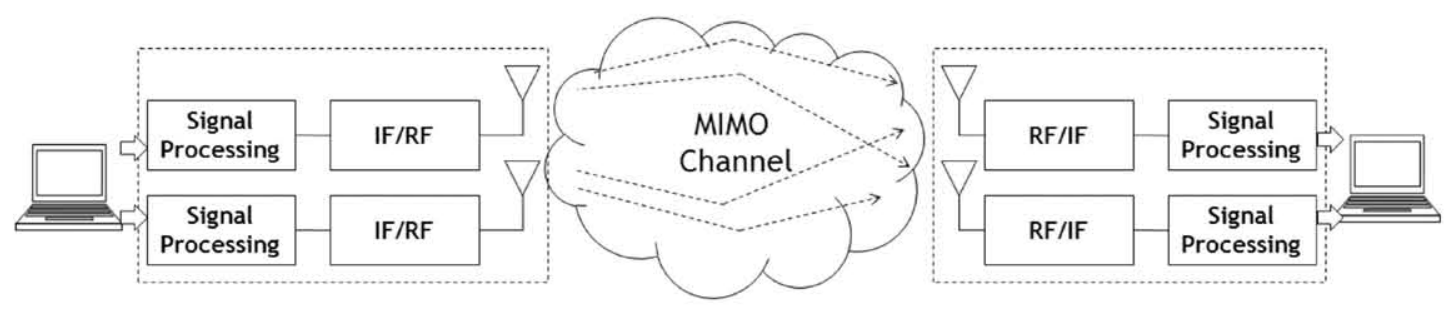

Fig. 1. General scheme of the MIMO testbed.

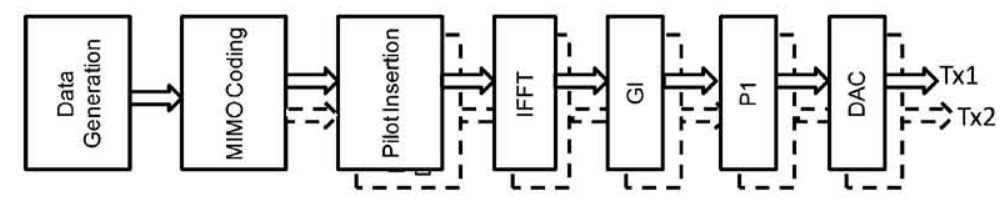

Fig. 2. Block diagram of the transmitter.

TABLE I

MIMO ENCODER WITH THE TRANSMITTED SYMBOLS

\begin{tabular}{|c|c|c|}
\hline subcarrier & Tx antenna 1 & Tx antenna 2 \\
\hline$k_{i}$ & $s_{1}$ & $-s_{2}^{*}$ \\
$k_{i+1}$ & $s_{2}$ & $s_{1}^{*}$ \\
\hline
\end{tabular}

channel estimation has been proposed in this paper, which has been tested by the corresponding simulations and finally, with measurements.

The paper is organized as follows: Section II describes the general architecture of the MIMO testbed with the different modules: signal processing (detailing the structure of the used signals in the testbed and the software-defined-radio platform used), radio frequency (RF) and antenna array. Section III explains the final integration of the testbed. Finally, Section IV comments on the measurements carried out with the testbed and Section V summarizes the conclusions.

\section{General Architecture of the MiMO TestBed}

The general architecture of the testbed is depicted in Fig. 1 where two antennas can be placed at the transmitter and the receiver side. The DVB-T2 signals are generated off-line in a PC (e.g. using Matlab) and then they are sent to the Software-Defined-Radio (SDR) platform. This platform receives the signals and transmits them in real-time and at Intermediate Frequency (IF) to the RF module. Finally, signals are upconverted to RF frequency, amplified, filtered and then transmitted to the radio channel through the antenna array. In the receiver, the signals are captured by the antenna array and downconverted, amplified and filtered by the RF module. Finally, the SDR realizes the synchronization and FFT demodulation before sending the signals to the PC. The main advantage of the system presented in this paper related to a channel sounder lies in the fact that this system generates real DVB-T2 signals and calculates the MIMO radio channel based on DVB-T2 pilot subcarrier patterns.

\section{A. Signal Processing Module}

The most complex part of the testbed is the signal processing module, since it supports all the digital to analog and analog to digital conversions (DAC and ADC) and the processing of the OFDM signal with the synchronization and the use of the
TABLE II

MAIN PARAMETERS OF THE TESTBED

\begin{tabular}{|c|c|c|}
\hline Parameter & Symbol & Value \\
\hline FFT Mode & - & $2 \mathrm{~K}$ \\
Modulation & - & QPSK, 16 or 64QAM \\
Sampling frequency & $F s$ & $9.1429 \mathrm{MHz}$ \\
Useful symbol time & $T u$ & $2048 / F s=224 \mu \mathrm{s}$ \\
Guard time & $T g$ & $T s / 8=28 \mu \mathrm{s}$ \\
Symbol time & $T s$ & $252 \mu \mathrm{s}$ \\
Bandwidth & $B W$ & $8 \mathrm{MHz}$ \\
Data subcarriers & $N_{d}$ & 1878 \\
\hline
\end{tabular}

Fast Fourier Transform (FFT). In the following paragraphs the transmitted DVB-T2 signals and SDR platform are explained.

1) DVB-T2 Signal: The DVB-T2 frame structure is divided in three different parts [15]. The first one is the P1 symbol which is used to do a faster detection and frequency synchronization. Then, the P2 symbol is transmitted to indicate the type of encoders and data configuration of the data symbols. However, for the sake of simplicity in this testbed the P2 symbol is removed. Finally the data symbols are sent with the user data and pilots for the channel estimation.

The block diagram of the transmitter is shown in Fig. 2. Data are generated and passed to the MIMO encoder which applies the distributed Alamouti system to transmit the signals by each antenna and then to recover the data symbols in the receiver. The Alamouti scheme [16] is modified with the scheme detailed in Table I.

After that, the continual and scattered pilots are inserted to estimate the channel at the receiver. Then, the Inverse-FFT (IFFT) is computed and the Guard Interval (GI) is inserted in order to avoid inter-symbol interference (ISI) due to the channel delay spread. The cyclic-prefix removes the ISI and converts the convolution between transmitted symbols and channel into a circular convolution. Finally, the P1 symbol is inserted and the two signals are converted from digital to analog and sent to RF module.

On the other hand, in Table II the most important parameters of the system are detailed as a summary.

2) SDR Platform: For the real-time process, two XtremeDSP boards based on the BenADDA module of Nallatech have been used: one for the transmitter and one for the receiver. Each board 


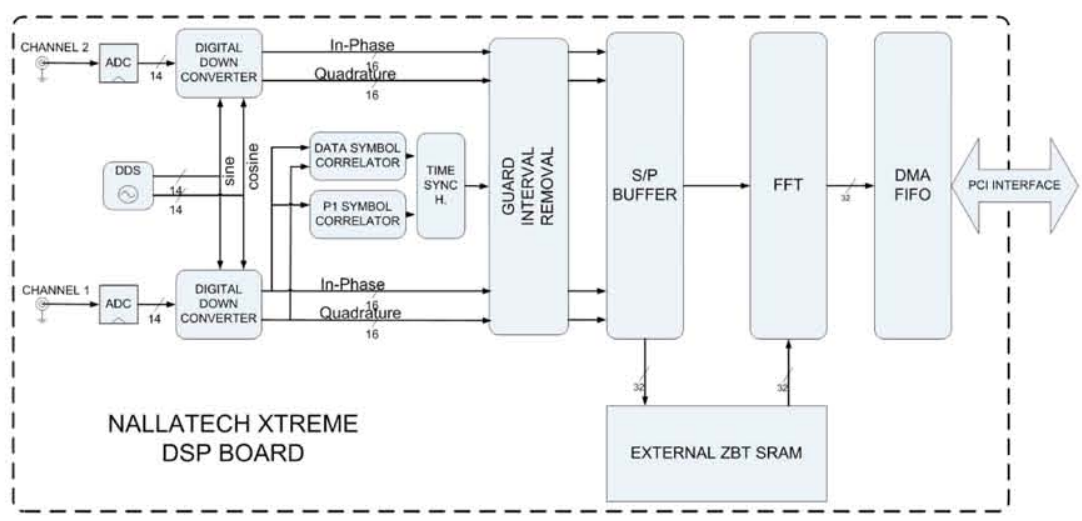

Fig. 4. Architecture of SDR receiver.

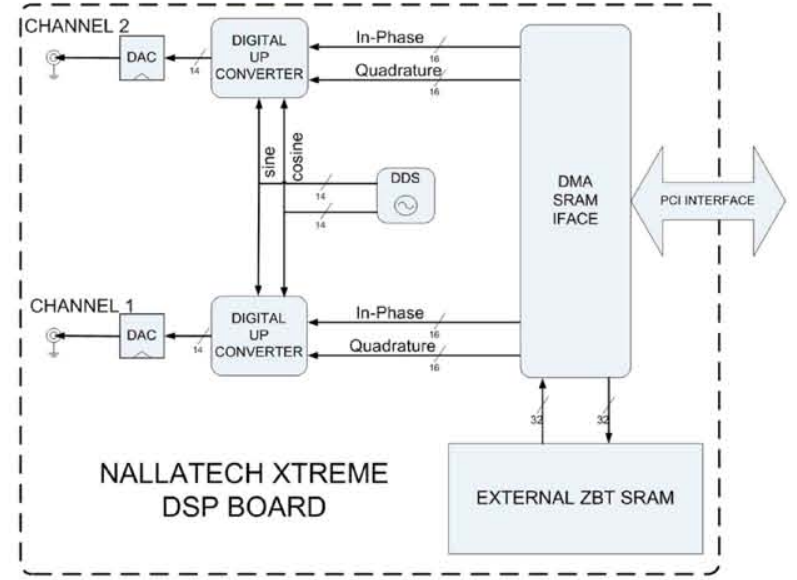

Fig. 3. Architecture of SDR transmitter.

has a FPGA VirteX II Pro V2P30 and 4 MBytes of SRAM memory with $2 \mathrm{DACs}$ of $160 \mathrm{MHz}$ and 2 ADCs of $105 \mathrm{MHz}$ [17].

Fig. 3 shows the architecture of the transmitter. The DVB-T signals are generated in off-line mode and are sent then to the board via DMA through the PCI bus. The DMA SRAM IFACE module stores them in the board's external memory ZBT SRAM. The maximum capacity is 512 samples per channel. Once the data write cycle is finished, the same module reads them from the memory and extracts them in a continuous and cyclic way. The I/Q data streams of each channel go to Digital Up Converter module, where the signals are interpolated by a factor of 10 and are upconverted to an IF of $36 \mathrm{MHz}$. The data are sent to DACs, which operate at a frequency of $91428571 \mathrm{~Hz}$. This frequency is selected for being 10 times the inverse of the sample period of a $8 \mathrm{MHz}$ channel, which according to the DVB-T2 standard [15] is $\mathrm{T}=7 / 64 \mu \mathrm{s}$.

Fig. 4 represents the main blocks of receiver subsystem. The signal from channel 1 is used to obtain the synchronism in time domain of the received symbols. The synchronization algorithm is based on the correlation of the cyclic prefix of OFDM symbols [18]. Then, the signals go to the Time Synch module which generates the synchronization signal, which allows the OFDM symbols to be obtained in the next module, named Guard Interval Removal. Finally, the FFT is computed to recover the block of data symbols.

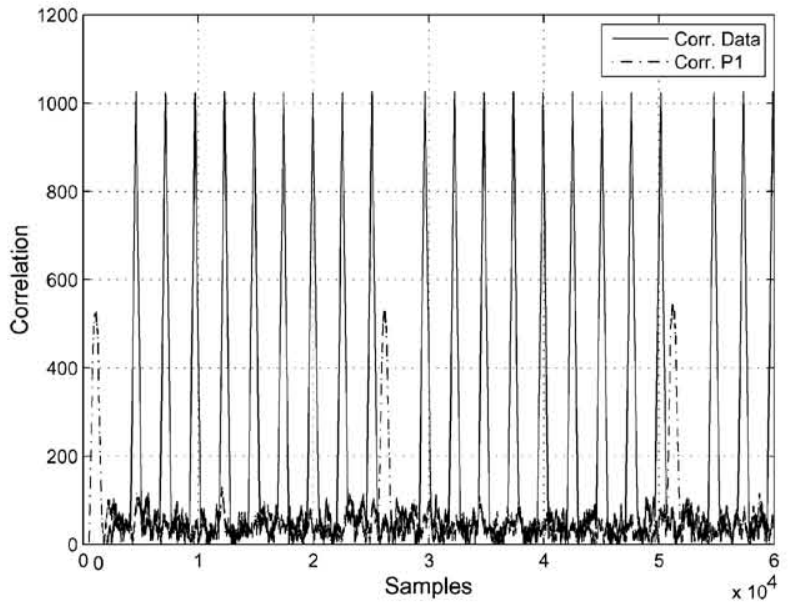

Fig. 5. Correlation of received signals. Dash line represents the correlation with the P1 symbol and solid line shows the autocorrelation with the data symbol.

In order to estimate the beginning of the frame, the signal passes through a correlator with the P1 symbol. Fig. 5 represents the correlation of a frame made up from a P1 symbol and 9 data symbols. Thus, the receiver takes the signals from the RF module and in real-time it processes them and gives as output the signals after having been converted by FFT. Then, a postprocessing module estimates the MIMO channel and saves the demodulate signals.

\section{B. RF Module}

The RF module consists of two chains with plug and coaxial commercial components, specially from Mini-Circuits. The design has been carried out taking into account the required power levels to perform outdoor/indoor measurements (+2 W RMS).

In the transmitter, the RF stage receives the signal from signal processing module and upconverts it from IF (36 MHz) to RF (594 MHz). Then, the signal is filtered and amplified (by the amplifier model LDU05M-R), with a transmitted power of $+2 \mathrm{~W}$ RMS. In order to upconvert the signals of both branches 1 and 2, a direct digital synthesizer (DDS) is used.

In the receiver, the signals are received from antenna ports and then are amplified by the Low Noise Amplifier (LNA) ZRL$115 \mathrm{LN}$ (with a typical noise figure of $1.1 \mathrm{~dB}$ ), filtered and downconverted to IF. In this case, a voltage controlled attenuator is placed to adapt the received signal power to the best range of 


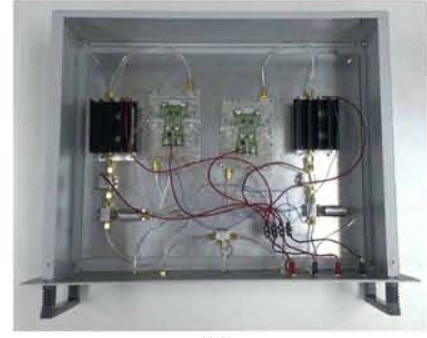

(a)

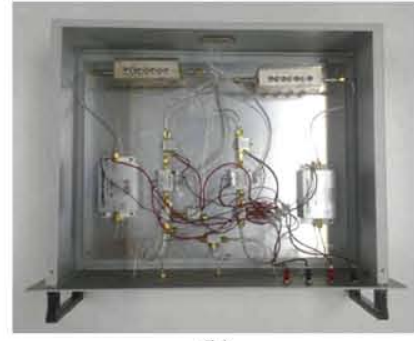

(b)
Fig. 6. RF implementation: (a) Tx; (b) Rx.

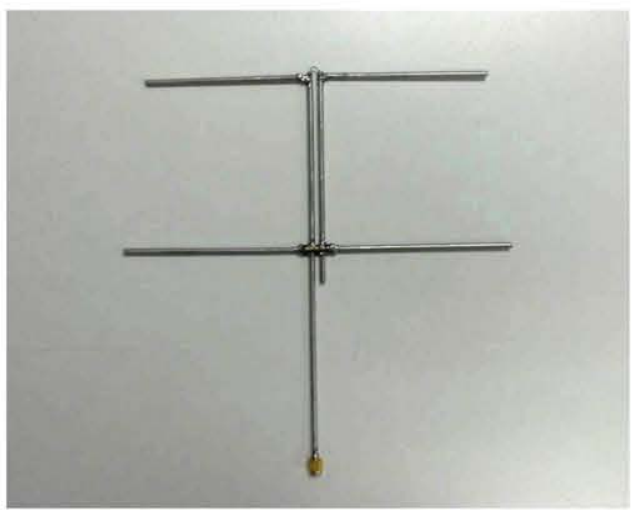

Fig. 7. Designed radiating element with a reflector and a $\lambda / 2$ dipole.

levels. The variation of the attenuator is from $3 \mathrm{~dB}$ to $38 \mathrm{~dB}$ in steps of $5 \mathrm{~dB}$.

Fig. 6 shows the final implementation of the RF modules, for the transmitter and the receiver, both based on coaxial and commercial components.

\section{Antenna Array Module}

The MIMO testbed has two antennas at the transmitter and two at the receiver, respectively. The antennas have been designed for the testbed to work at $594 \mathrm{MHz}$. Typically, the antenna used in DVB-T systems for the reception is a Yagi antenna, which consist in a $\lambda / 2$ dipole with one reflector and many directors, although other antennas can be found in the literature [19]. In order to simplify the antenna design, for the MIMO testbed, the radiating element is a dipole with one reflector element, as Fig. 7 shows. The length of the dipole is approximately $\lambda / 2$, which in this case is $22 \mathrm{~cm}$.

The reflection coefficient of the transmitter and receiver antennas is depicted in Fig. 8. It shows that all the implemented antennas have a good matching at desired frequency band, obtaining a reflection coefficient lower than $-17 \mathrm{~dB}$. Furthermore, the radiation pattern is presented in Fig. 9, where the Cross-Polarization Discrimination (XPD) is higher than $25 \mathrm{~dB}$ in the maximum direction of $\theta=0^{\circ}$.

\section{Channel Estimation}

One of the key aspects in MIMO is the channel estimation, since the performances of MIMO systems depend on the accuracy of the estimated channel matrix.

Due to the selection of $2 \mathrm{~K}$ mode of FFT and a GI of $1 / 8$, the DVB-T2 standard [15] proposes eight different patterns for

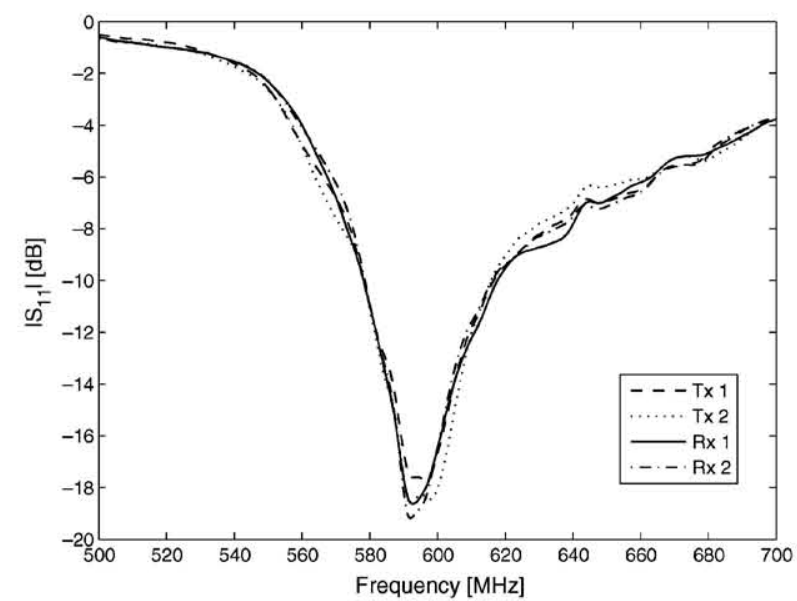

Fig. 8. Reflection coefficient of the antennas.

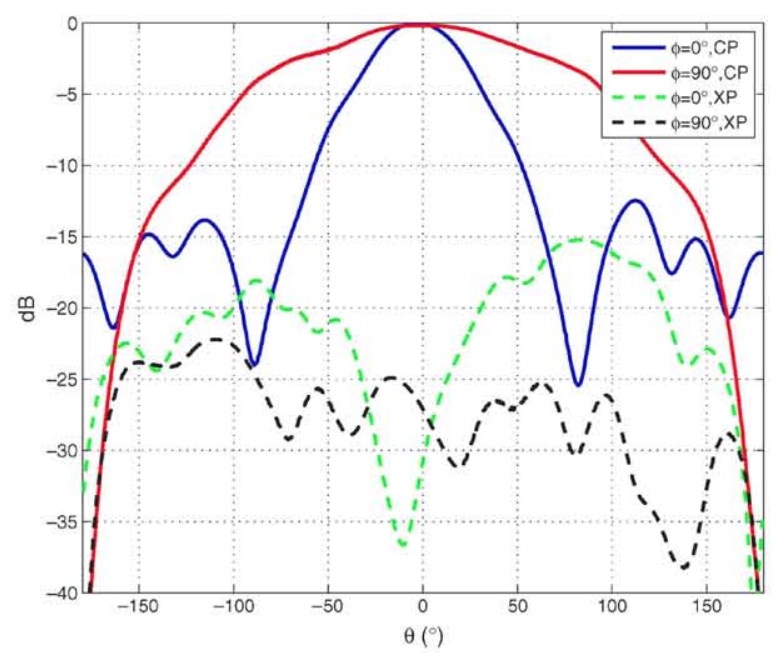

Fig. 9. Measured radiation pattern of an antenna.

scattering pilots, depending on the size of FFT and the GI. In the case of using a $2 \mathrm{~K}$ FFT and $1 / 8$ of GI, the corresponding pattern for scattering pilots for MISO is the PP1 pattern.

The scattered pilots, for each OFDM symbol are placed each 12 subcarriers along the frequency axis. Attending to the temporal axis, the pilots start in the first subcarrier and the initial point is shifted in 2 subcarriers for the following 3 OFDM symbols, as Fig. (10a) and (10b) show for antenna 1 and 2, respectively. Thus, the pilots structure is organized in 4-symbol blocks in time domain and it depends on the used antenna. In antenna 2 case (Fig. (10b)), the corresponding pilots to symbols 1 and 3 are inverted to distinguish the transmitter antenna. Moreover, it is worth mentioning here that the scattering pilots are generated according to a Pseudorandom Binary Sequence (PRBS) [15].

Thus, if $\mathbf{X}_{k}$ represents the transmitted symbols vector for both antennas for the $k$-subcarrier, the received vector $\mathbf{R}_{k}$ is given by

$$
\mathbf{R}_{k}=\mathbf{H}_{k} \mathbf{X}_{k}+\mathbf{N}_{k}
$$

where $\mathbf{H}_{k}$ represents the MIMO channel matrix and $\mathbf{N}_{k}$ represents the noise vector for the two receivers, both for the $k$-subcarrier. Since the testbed has two antennas in the transmitter and 


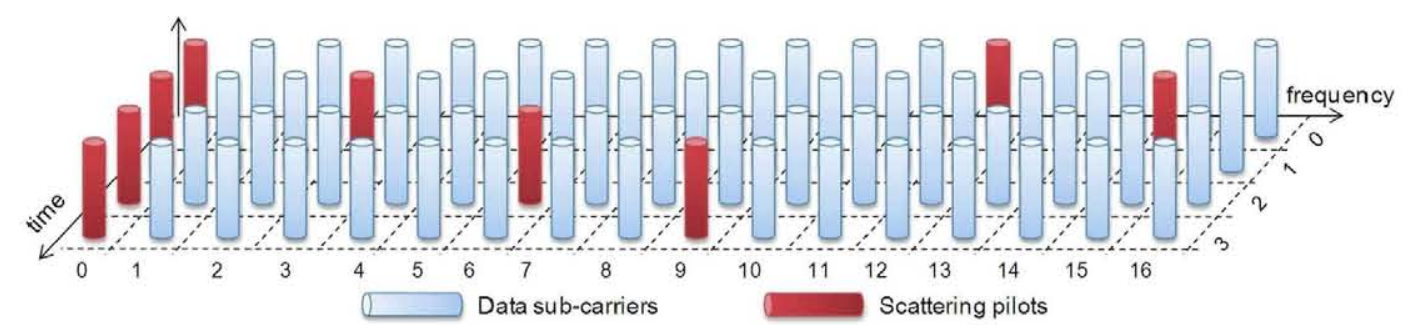

(a)

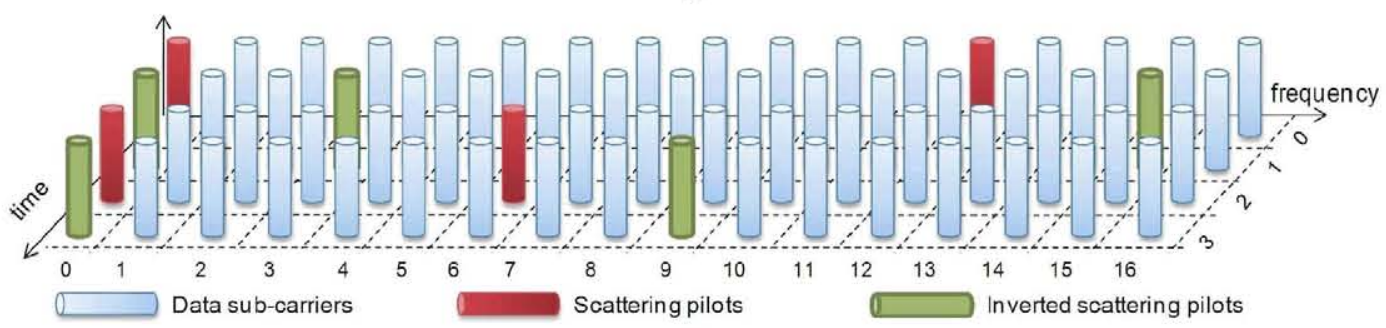

(b)

Fig. 10. Scattered pilots distribution. (a) Antenna 1; (b) Antenna 2.

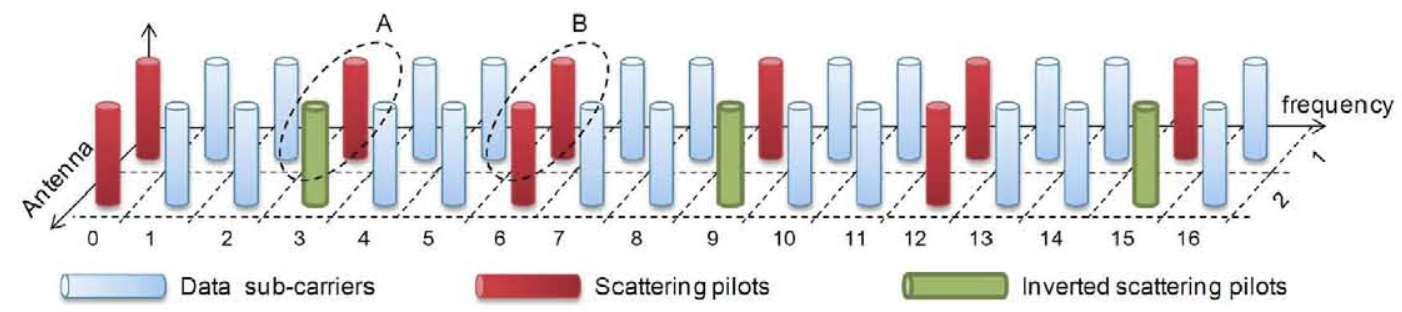

Fig. 11. Scattered pilots association for antennas 1 and 2 .

another two in the receiver, the MIMO channel matrix is represented by

$$
\mathbf{H}_{k}=\left[\begin{array}{ll}
h_{11, k} & h_{12, k} \\
h_{21, k} & h_{22, k}
\end{array}\right]
$$

where $h_{i j, k}$ represents the channel response between receiver antenna $i$ and transmitter antenna $j$ in the $k$-subcarrier.

Some channel estimators have been proposed recently for DVB-T systems [20], [21], however very few works have been found using MIMO. In the literature a MIMO channel estimator for DVB-T2 has not been yet proposed, so in this section an original scheme is presented to estimate the channel for the MIMO case.

First of all, the channel is assumed not to vary in time domain for four data symbols, which is a similar assumption to other channel estimators for DVB-T [22]-[25]. Therefore, the pilots are gathered in groups of four OFDM data symbols (in frequency domain) in only one symbol, obtaining one pilot subcarrier out of three subcarriers. Fig. 11 depicts the association for both antennas.

The received signals at the antennas 1 and $2, r_{1, k}$ and $r_{2, k}$, respectively, for the $k$-subcarrier are given by

$$
\begin{aligned}
& r_{1, k}=x_{1, k} \cdot h_{11, k}+x_{2, k} \cdot h_{12, k}+n_{1, k} \\
& r_{2, k}=x_{1, k} \cdot h_{21, k}+x_{2, k} \cdot h_{22, k}+n_{2, k}
\end{aligned}
$$

where $x_{i, k}$ represents the pilot signal transmitted by antenna $i$, and $n_{i, k}$ is the noise contribution in antenna $i$. From this point two cases are considered to estimate the channel. The first one is the case in which the pilots of both antennas are scattered pilots for a given subcarrier. This case is marked with $\mathrm{A}$ in Fig. 11. In the other case, the pilot for one antenna is a scattered pilot and for the other antenna is an inverted scattered pilot, for a given subcarrier. This is case B in Fig. 11.

Thus, for case $A$ the received signals, from (1) and taking into account the inverted scattering pilots, are:

$$
\begin{aligned}
r_{1, k}^{a} & =x_{1, k}^{a} \cdot h_{11, k}-x_{1, k}^{a} \cdot h_{12, k}+n_{1, k}^{a} \\
& =x_{1, k}^{a}\left(h_{11, k}-h_{12, k}\right)+n_{1, k}^{a} \\
r_{2, k}^{a} & =x_{1, k}^{a} \cdot h_{21, k}-x_{1, k}^{a} \cdot h_{22, k}+n_{2, k}^{a} \\
& =x_{1, k}^{a}\left(h_{21, k}-h_{22, k}\right)+n_{2, k}^{a}
\end{aligned}
$$

and for case B

$$
\begin{aligned}
r_{1, k}^{b} & =x_{1, k}^{b} \cdot h_{11, k}+x_{1, k}^{b} \cdot h_{12, k}+n_{1, k}^{b} \\
& =x_{1, k}^{b}\left(h_{11, k}+h_{12, k}\right)+n_{1, k}^{b} \\
r_{2, k}^{b} & =x_{1, k}^{b} \cdot h_{21, k}+x_{1, k}^{b} \cdot h_{22, k}+n_{2, k}^{b} \\
& =x_{1, k}^{b}\left(h_{21, k}+h_{22, k}\right)+n_{2, k}^{b}
\end{aligned}
$$




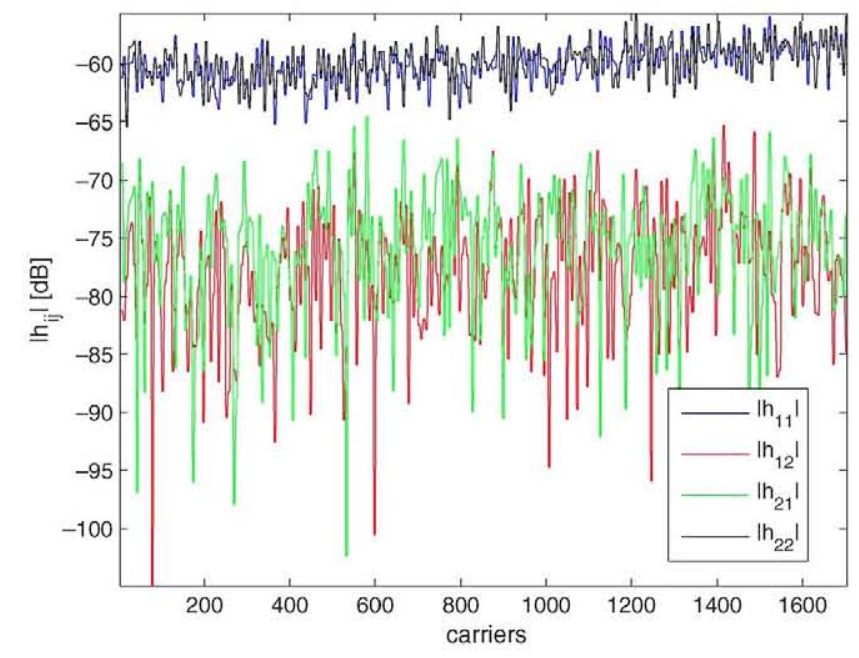

Fig. 12. Modulus of channel estimation for a TX-RX test.

Operating with the obtained signals from (5) to (8), channel coefficients can be estimated as

$$
\begin{aligned}
& \tilde{h}_{11, k}=\frac{r_{1, k}^{a}+r_{1, k}^{b} \frac{x_{1, k}^{a}}{x_{1, k}^{b}}}{2 x_{1, k}^{a}} \\
& \tilde{h}_{12, k}=\frac{r_{1, k}^{a}-r_{1, k}^{b} \frac{x_{1, k}^{a}}{x_{1, k}^{b}}}{-2 x_{1, k}^{a}} \\
& \tilde{h}_{21, k}=\frac{r_{2, k}^{a}+r_{2, k}^{b} \frac{x_{1, k}^{a}}{x_{1, k}^{b}}}{2 x_{1, k}^{a}} \\
& \tilde{h}_{22, k}=\frac{r_{2, k}^{a}-r_{2, k}^{b} \frac{x_{1, k}^{a}}{x_{1, k}^{b}}}{-2 x_{1, k}^{a}}
\end{aligned}
$$

And the estimated MIMO channel matrix is

$$
\tilde{\mathbf{H}}_{k}=\left[\begin{array}{ll}
\tilde{h}_{11, k} & \tilde{h}_{12, k} \\
\tilde{h}_{21, k} & \tilde{h}_{22, k}
\end{array}\right]
$$

To evaluate the presented algorithm for channel estimation, a test has been realized by connecting the signal processing module of the transmitter to the receiver one in IF. In order to achieve this, the output of branch 1 of the transmitter was linked to the input 1 of the receiver and the transmitter branch 2 to the receiver branch 2 by a $60 \mathrm{~dB}$ attenuator. In this situation, the channel estimator should give a modulus of $h_{11}$ and $h_{22}$ higher than the others and equal to $-60 \mathrm{~dB}$, as Fig. 12 shows. Moreover, it should give a linear phase variation for $h_{11}$ and $h_{22}$ cases and non linear values for $h_{12}$ and $h_{21}$, since they are not connected. Fig. 13 represents the estimated phase of the MIMO channel measured for the four matrix elements. The phase for the $h_{11}$ and $h_{22}$ has a linear behavior due to the fact that a frequency error is introduced by using different oscillators with slightly different frequencies for transmission and reception in IF.

\section{INTEGRATION}

Once the different modules of the transmitter and receiver were implemented and tested to work properly, they were integrated. In Fig. 14 the transmitter and receiver are illustrated. The

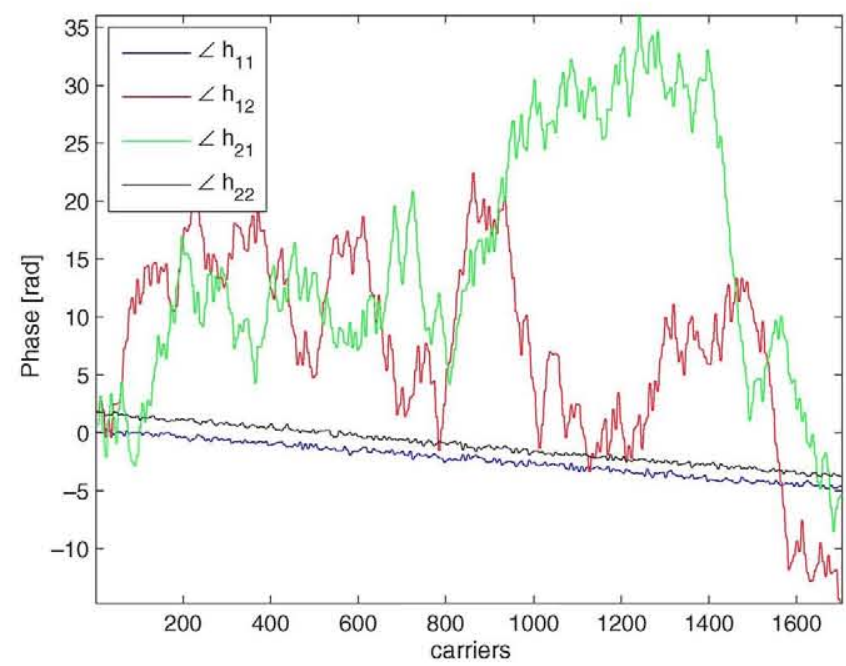

Fig. 13. Phase of channel estimation for a TX-RX test.

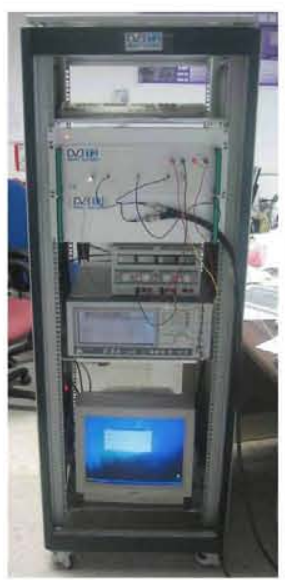

(a)

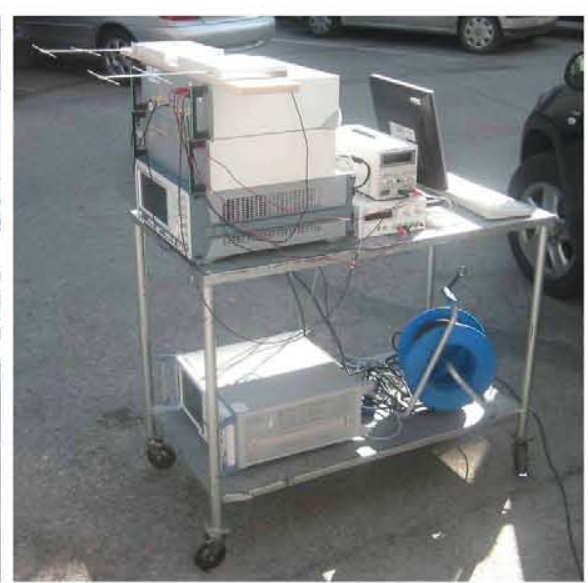

(b)
Fig. 14. Transmitter and receiver integration: (a)Tx; (b) Rx.

transmitter is placed on a $19^{\prime \prime}$ rack and the receiver is mounted on a mobile platform in order to measure different environments.

As in any real system, implementation issues such as frequency errors that have been introduced by using different local oscillators internal clocks at each side of the radio link appear. In general, all the errors can be represented as

$$
\mathbf{R}_{k}=\mathbf{X}_{k} \mathbf{H}_{k} \cdot e^{j\left(2 \pi k \frac{T_{d}}{T_{u}}+2 \pi k \frac{\Delta l(n)}{T_{u}}+\phi_{0}+2 \pi k \Delta f T_{0}\right)}+\mathbf{I}_{k}+\mathbf{N}_{k}
$$

where $T_{d}$ represents the symbol temporal offset, $\Delta t$ the sampling temporal offset, $\phi_{0}$ the phase offset, $\Delta f$ the frequency offset and $\mathbf{I}_{k}$ is the ICI (Inter-Carrier Interference) due to frequency offset for the $k$-carrier. These errors must be taken into account and have been mitigated in the signal processing module by means of correlations and calculations of the received phases.

\section{Measurements}

Once the MIMO testbed has been realized, a measurement campaign has been carried out in order to evaluate the enhancement obtained by using the MIMO scheme. 


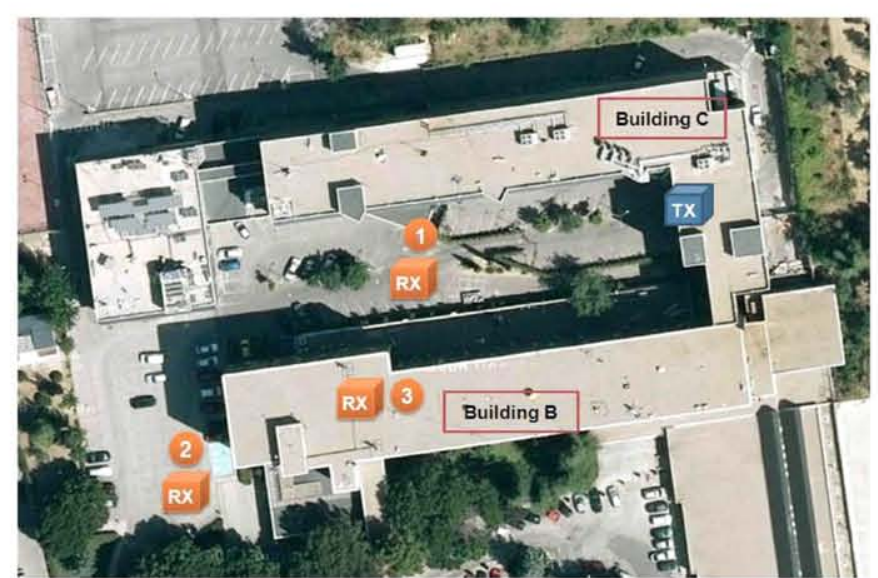

Fig. 15. Top view of the Tx and Rx positions.

The measurements were carried out at the Telecommunication Engineering School, at Universidad Politécnica de Madrid, Spain. The transmitter was situated on the rooftop of building $\mathrm{C}$ with a spacing between elements of $1 \lambda$. Fig. 15 depicts the topview of the measurement locations. In order to measure several scenarios, the receiver was located in three different positions (but several measurements were taken moving the receiver in several locations for the same position). In position 1, the receiver was placed in the parking area of the building in LoS (Line of Sight). The receiver for NLoS (Non Line of Sight) was situated in position 2 , in the car park of the next building. Finally, for outdoor to indoor scenario, the receiver was located in the third floor of the building, as Fig. 15 shows. For DVB-T, the use of orthogonal polarization has been employed to other purposes, such as mitigate impulse noise [26], but few measurements are applied for MIMO.

Thus, due to the lack of measurements of MIMO channels using polarization diversity, for each scenario, three different polarization schemes were evaluated: $\mathrm{HH}, \mathrm{HV}$ and $\mathrm{VV}$, where $\mathrm{H}$ and $\mathrm{V}$ means horizontal and vertical polarization for antennas 1 and 2 both in the transmitter and the receiver, respectively. The same configuration of polarization diversity has been used both for the transmitter and receiver arrays, since a future DVB-T2 user has to receive the same polarization scheme as the transmitter. The tilt was $20^{\circ}$ and the spacing between elements was fixed in $1 \lambda$ to achieve a better orthogonality (or less correlation) among the MIMO subchannels. Moreover, the input power was $2 \mathrm{~W}$ at each port of the transmitter antennas.

\section{A. MIMO Channel}

The first step for evaluating the MIMO channel is to normalize channel gain. After channel estimation, the channel power is calculated for all the measured cases. The channel power is averaged over all frequencies $f_{v}$ and temporal snapshots $t_{u}$ and is calculated by

$$
P_{m}=\frac{1}{N_{T} N_{f}} \sum_{t_{u}=1}^{N_{T}} \sum_{f_{v}=1}^{N_{f}} P\left(t_{u}, f_{v}\right)
$$

where $N_{T}$ represents the number of OFDM symbols (100 in this case), $N_{f}$ indicates the number of subcarriers, which is 2048 due to the use of a FFT of $2 \mathrm{~K}$, since for the power calculation
TABLE III

COMPARISON OF MEASURED MEAN POWER

\begin{tabular}{|c|c|c|c|}
\hline Measured & \multicolumn{3}{|c|}{ Mean channel power (dB) } \\
\cline { 2 - 4 } scenario & HH & HV & VV \\
\hline 1 - Outdoor LoS & -63.5 & -62.8 & -63.2 \\
2 - Outdoor NLoS & -81.1 & -81.8 & -73.7 \\
3 - Indoor NLoS & -67.9 & -68.8 & -69.5 \\
\hline
\end{tabular}

TABLE IV

COMPARISON OF MEASURED XPD

\begin{tabular}{|l|c|}
\hline Measured scenario & XPD $(\mathrm{dB})$ \\
\hline 1 - Outdoor LoS & 11.1 \\
2 - Outdoor NLoS & 10.3 \\
3 - Indoor NLoS & 7.9 \\
\hline
\end{tabular}

all the subcarriers are taken into account (pilots and data); and $P$ represents the mean power in the measured point and is given by

$$
P\left(t_{u}, f_{v}\right)=\frac{\left\|\mathbf{H}\left(t_{u}, f_{v}\right)\right\|_{F r o}^{2}}{M_{T} M_{R}}
$$

where $M_{R}$ and $M_{T}$ represent the number of receiver and transmitter antennas, respectively.

Table III shows the comparison of mean power for each scenarios as a function of antenna polarization. It is shown in LoS scenarios, where the received power is increased. However, in indoor case the power only is reduced in $6 \mathrm{~dB}$ because the distance between the transmitter and receiver is about $23 \mathrm{~m}$ smaller than in the case 3 .

Moreover, the XPD has been calculated for all the cases as [27]

$$
\begin{aligned}
& X_{V}=\mathcal{E}\left\{\left|h_{V V}\right|^{2}\right\} / \mathcal{E}\left\{\left|h_{H V}\right|^{2}\right\} \\
& X_{H}=\mathcal{E}\left\{\left|h_{H H}\right|^{2}\right\} / \mathcal{E}\left\{\left|h_{V H}\right|^{2}\right\}
\end{aligned}
$$

where $\mathcal{E}$ is the mathematical expectation and XPD is calculated as the average of $X_{V}$ and $X_{H}$. Results in Table IV show a mean XPD of $10 \mathrm{~dB}$.

\section{B. MIMO Capacity}

Besides the diversity gain, the other important feature of MIMO channels is the increase of the channel capacity. In the case of DVB-T2, the transmitter does not know the Channel State Information (CSI), and the MIMO capacity can be calculated from [3]

$$
C=\sum_{k=1}^{N_{f}} \log _{2}\left[\operatorname{det}\left(\mathbf{I}_{M_{R}}+\frac{\rho}{M_{T}} \mathbf{H}_{k} \mathbf{H}_{k}^{H}\right)\right] \mathrm{bps} / \mathrm{Hz}
$$

where $k$ is the given subcarrier, $N$ represents the total number of subcarriers and $\rho$ is the Signal to Noise Ratio (SNR). In this paper, the measurements have been realized with $M_{T}=M_{R}=$ 2 . This expression is valid under some certain conditions, which are the perfect knowledge of the channel at the receiver, the assumption of a Rayleigh channel, and the same transmitted power by each antenna, so the approximation is assumable for the results presented in this paper. On the other hand, the channel matrices are calculated using the normalized channel matrices to compare all the scenarios. 


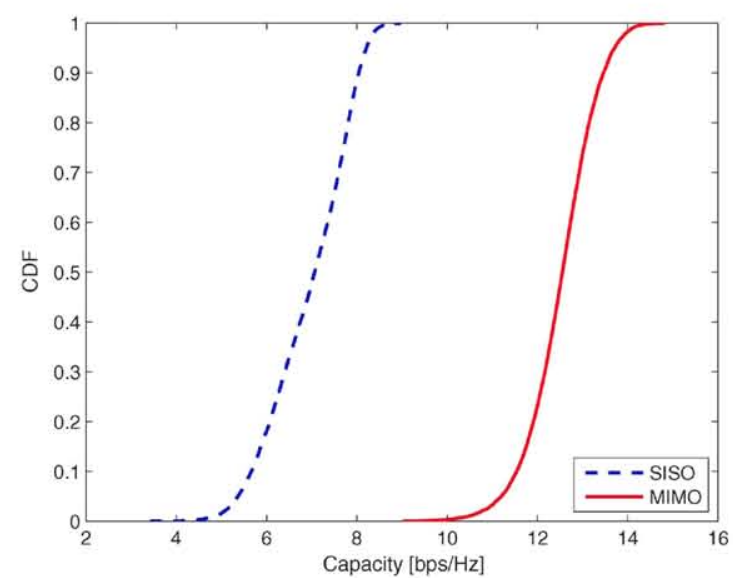

Fig. 16. Comparison of CDF of capacity with $1 \times 1$ and $2 \times 2$ in outdoor LoS case with polarization diversity.

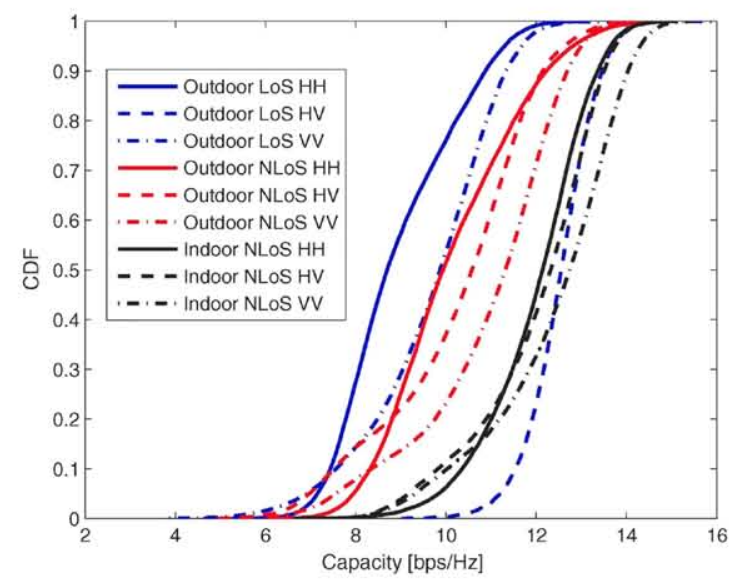

Fig. 17. CDF of capacity for all the measured scenarios.

TABLE V

COMPARISON OF CAPACITY FOR ALL THE MEASUREd CASES

\begin{tabular}{|c|c|c|c|c|}
\hline \multicolumn{2}{|c|}{$\begin{array}{c}\text { Measured } \\
\text { scenario }\end{array}$} & $\begin{array}{c}C_{\text {out }} \\
\text { (bps/Hz) }\end{array}$ & $\begin{array}{c}C_{\text {mean }} \\
\text { (bps/Hz) }\end{array}$ & $\begin{array}{c}\sigma_{c} \\
\text { (bps/Hz) }\end{array}$ \\
\hline & $\mathrm{HH}$ & 7.46 & 8.99 & 1.30 \\
1 - Outdoor LoS & $\mathrm{HV}$ & 11.5 & 12.54 & 0.76 \\
& $\mathrm{VV}$ & 7.53 & 9.71 & 1.45 \\
\hline \multirow{3}{*}{ 2 - Outdoor NLoS } & $\mathrm{HH}$ & 8.32 & 10.18 & 1.50 \\
& $\mathrm{HV}$ & 7.47 & 10.28 & 1.71 \\
& $\mathrm{VV}$ & 8.31 & 10.99 & 1.62 \\
\hline \multirow{3}{*}{3 - Indoor NLoS } & $\mathrm{HH}$ & 10.36 & 12.01 & 1.17 \\
& $\mathrm{HV}$ & 9.79 & 12.03 & 1.38 \\
& $\mathrm{VV}$ & 10.00 & 11.72 & 1.25 \\
\hline
\end{tabular}

With the aim of comparing the capacity of SISO and MIMO, Fig. 16 represents the cumulative distribution function of the capacity calculated for the $\operatorname{LoS}$ case with polarization diversity. The capacity increases in the $2 \times 2$ case.

On the other hand, Fig. 17 shows the comparison of all the cases by using different polarization schemes. The highest values of capacity are obtained for indoor NLoS, followed by outdoor $\mathrm{NLoS}$ and the, outdoor LoS, except when polarization diversity (HV) is used in LoS case, since the obtained outage capacity at $10 \%$ is the highest.

To sum up all the capacity values, Table $\mathrm{V}$ shows the comparison of the capacities for outage (10\%), mean and the standard deviation. Attending to outage capacity, the highest values are obtained for the outdoor LoS HV case and the indoor situations for all the polarization schemes.

\section{CONCLUSIONS}

A novel $2 \times 2$ MIMO testbed has been designed and implemented for the future digital television system DVB-T2. The testbed is based on software radio platforms where the signal processing is implemented according to the standard. The testbed has been designed to carry out measurements at the frequency of $594 \mathrm{MHz}$. Moreover, a new channel estimation scheme has been proposed for MIMO DVB-T2 systems. A measurement campaign has been conducted in outdoor and outdoor to indoor scenarios. Results show the importance of using multiple antennas at each side of the radio link for increasing the capacity of the underlying radio transmission system. Moreover, it has also been shown that polarization diversity provides an additional capacity gain especially for outdoor $\mathrm{LoS}$ cases (up to $4 \mathrm{bps} / \mathrm{Hz}$ in outage capacity). This can increase the number of SDTV or HDTV programs since it enhances the spectral efficiency. The measurements carried out were taken for several positions in fixed environments, so as a future work, it will be very interesting the fact of placing the receiver in a vehicle and taking measurements in movement to analyse the use of MIMO diversity in DVB-T channels.

\section{ACKNOWLEDGMENT}

The authors would like to thank Ministerio de Industria, Turismo y Comercio for the support in the FURIA 2 project.

\section{REFERENCES}

[1] Digital Video Broadcasting, 2003 [Online]. Available: http://www. dvb.org

[2] G. Foschini and M. Gans, "On limits of wireless communications in a fading environment when using multiple antennas," Wireless Personal Communications, vol. 6, pp. 311-335, Mar. 1998.

[3] E. Telatar, "Capacity of multi-antenna Gaussian channels," Eur. Trans. Telecomm. ETT, vol. 10, no. 6, pp. 585-596, Nov. 1999.

[4] S. Caban, R. Langwieser, C. Mehlführer, E. Aschbacher, W. Keim, G. Maier, B. Badic, M. Rupp, and A. Scholtz, "Design of a flexible and scalable $4 \times 4$ MIMO testbed," in Digital Signal Processing Workshop, 2004 and the 3rd IEEE Signal Processing Education Workshop. 2004 IEEE 11th, Sep. 2004, pp. 178-181.

[5] R. M. Rao, W. Zhu, S. Lang, C. Oberli, D. Browne, J. Bhatia, J.-F. Frigon, J. Wang, P. Gupta, and H. Lee, "Multi-antenna testbeds for research and education in wireless communications," IEEE Communications Magazine, vol. 42, no. 12, pp. 72-81, Dec. 2004.

[6] D. Chizhik, G. Foschini, M. Grans, and R. Valenzuela, "Keyholes, correlations and capacities of multielement transmit and receive antennas," IEEE Trans. Wireless Communications, vol. 1, no. 2, pp. 361-368, Apr. 2002.

[7] M. A. I. et al., "Georgia tech MIMO-OFDM link prototype," in Workshop on MIMO Implementation Aspects: IEEE Radio and Wireless Conference RAWCON, Atlanta, GA, Sep. 2004.

[8] J. Mitchell, P. Moss, and M. J. Thorp, "A dual polarisation MIMO broadcast TV system," in BBC, Research White Paper WHP 144, Dec. 2006.

[9] J. Boyer, P. Brown, K. Hayler, M. L. Garcia, J. Mitchell, P. Moss, and M. Thorp, "MIMO for broadcast-Results from a high-power UK trial," in BBC, Research White Paper WHP 157, Oct. 2007.

[10] C. Wang, G.-N. Sung, J.-Y. Liao, J. Chang, and R. Hu, "Handheld DVB-T digital TV with an automatic antenna selection method for mobile reception," in International Conference on Consumer Electronics, 2007. ICCE 2007. Digest of Technical Papers, Jan. 2007, pp. 1-2.

[11] C. Gómez-Calero, L. Garcia-Garcia, R. Martinez, and L. de Haro, "Comparison of antenna configurations in different scenarios using a wideband MIMO testbed," in IEEE Antennas and Propagation Society International Symposium 2006, Jul. 2006, pp. 301-304. 
[12] A. Hammons, J. Hampton, N. Merheb, and M. Cruz, "Cooperative MIMO field measurements for military UHF band in low-rise urban environment," in 5th IEEE Sensor Array and Multichannel Signal Processing Workshop, 2008. SAM 2008, Jul. 2008, pp. 122-126.

[13] R. Di Bari, M. Bard, Y. Zhang, K. Nasr, J. Cosmas, K.-K. Loo, R. Nilavalan, H. Shirazi, and K. Krishnapillai, "Laboratory measurement campaign of DVB-T signal with transmit delay diversity," IEEE Trans. Broadcasting, vol. 54, no. 3, pp. 532-541, Sep. 2008.

[14] T. Willink, "Characteristics of urban vehicular MIMO channels at different frequencies," in 3rd European Conference on Antennas and Propagation, Berlin, Germany, Mar. 2009.

[15] "Digital Video Broadcasting (DVB);Frame Structure Channel Coding and Modulation for a Second Generation Digital Terrestrial Television Broadcasting System (DVB-T2)," ETSI, Draft ETSI EN 302755 V1.1.1 (2008-04), 2008.

[16] S. Alamouti, "A simple transmit diversity technique for wireless communications," IEEE Journal on Selected Areas in Communications, vol. 16, pp. 1451-1458, Oct. 1998.

[17] Nallatech, [Online]. Available: http://www.nallatech.com

[18] J. van de Beek, M. Sandell, and P. Borjesson, "ML estimation of time and frequency offset in OFDM systems," IEEE Trans. Signal Processing, vol. 45 , no. 7 , pp. 1800-1805, Jul. 1997.

[19] S. Panagiotou, S. Mitilineos, T. Dimousios, and C. Capsalis, "A broadband, vertically polarized, circular switched parasitic array for indoor portable DVB-T applications at the IV UHF band," IEEE Trans. Broadcasting, vol. 53, no. 2, pp. 547-552, Jun. 2007.

[20] J. C. Lee, D. S. Han, and S. Park, "Channel estimation based on path separation for dvb-t in long delay situations," IEEE Trans. Consumer Electronics, vol. 55, no. 2, pp. 316-321, May 2009.

[21] A. Goljahani, N. Benvenuto, S. Tomasin, and L. Vangelista, "Superimposed sequence versus pilot aided channel estimations for next generation dvb-t systems," IEEE Trans. Broadcasting, vol. 55, no. 1, pp. 140-144, Mar. 2009.

[22] Y.-S. Lee, H.-N. Kim, and K. S. Son, "Noise-robust channel estimation for DVB-T fixed receptions," IEEE Trans. Consumer Electronics, vol. 53, no. 1, pp. 27-32, Feb. 2007.

[23] A. Palin and J. Rinne, "Symbol synchronization in OFDM system for time selective channel conditions," in Proceedings of The 6th IEEE International Conference on Electronics, Circuits and Systems, 1999, vol. 3, pp. 1581-1584.

[24] S.-H. Chen, W.-H. He, H.-S. Chen, and Y. Lee, "Mode detection, synchronization, and channel estimation for DVB-T OFDM receiver,' in IEEE Global Telecommunications Conference, 2003. GLOBECOM 'O3, Dec. 2003, vol. 5, pp. 2416-2420.

[25] R.-D. Fang and H.-P. Ma, "A DVB-T/H baseband receiver for mobile environments," in 2007 WSEAS International Conference on Circuits, Systems, Signal and Telecommunications, Queensland, Australia, Jan. 2007.

[26] P. Torio and M. Sanchez, "Mitigation of impulsive noise in digital video broadcasting terrestrial using orthogonal polarization reception," IEEE Trans. Consumer Electronics, vol. 55, no. 4, pp. 1798-1804, Nov. 2009.

[27] V. Anreddy and M. Ingram, "Capacity of measured Ricean and Rayleigh indoor MIMO channels at $2.4 \mathrm{GHz}$ with polarization and spatial diversity," in IEEE Wireless Communications and Networking Conference, 2006. WCNC 2006., Apr. 2006, vol. 2, pp. 946-951.

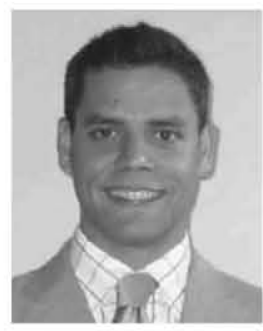

Carlos Gómez-Calero was born in Madrid, Spain, in 1981. He received the ingeniero técnico de telecomunicación degree, the ingeniero de telecomunicación degree and the Doctor ingeniero de telecomunicación degree (cum laude), from Universidad Politécnica de Madrid (UPM), Spain, in 2002, 2005 and 2009, respectively.

From 2003 he joined to the Radiation Group of the Signals, Systems and Radiocommunications department in UPM as a researcher. He has participated as a lecturer in several international courses about smart antennas. His research works has been published in international journals, conferences, patents and chapter of books. Currently he works in the European Space Agency (ESA). His research interests are in the areas of smart antennas, telecommunication systems, mobile and satellite communications.

Dr. Gomez-Calero received the award to "The Best Master Thesis in Radiocommunications" from Rohde \& Schwarz in 2003. He received in 2005 in Newport (USA) "The Best Student Paper Award" in 2005 AMTA Symposium. In 2007 he received the Foundation VODAFONE Award to the "Best Master Thesis in Advanced Mobile Communications", and the REsA Award to the "Best Paper in Antennas and Propagation" in XXII URSI Symposium.

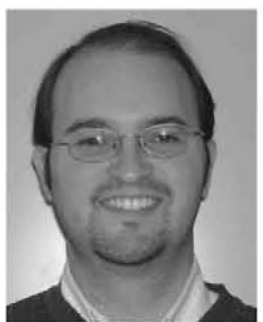

Luis Cuéllar Navarrete was born in Madrid, Spain in 1979. He received the BS degree in telecommunication engineering from Universidad Politécnica de Madrid, Spain (UPM), in 2006.

From 2004 he joined to the Radiation Group of the Signals, Systems and Radiocommunications department in UPM as a researcher. He participated in various investigation projects related to OFDM transmission systems, including an SFN repeater with echo-cancellation for DVB-T and DAB systems. His research interests include digital communications with emphasis on OFDM and algorithm synthesis for synchronization and equalization.

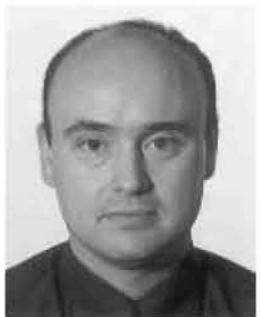

Leandro de Haro received the ingeniero de telecomunicación degree in 1986 and the Doctor Ingeniero de Telecomunicación degree (Apto cum laude) in 1992, both from the E.T.S.I. Telecomunicación, Departamento de Señales, Sistemas y Radiocomunicaciones; Universidad Politécnica de Madrid (UPM), Spain.

Since 1990, he has developed his professional career in the Departamento de Señales, Sistemas y Radiocomunicaciones as Professor Titular de Universidad in the signal theory and communications area. His research activity covers the following topics: antenna design for satellite communications (earth stations and satellite on board); study and design of satellite communication systems; and study and design of digital TV communication systems. He has been actively involved in several official projects and with private companies (national and international). He has also been involved in several European projects (RACE, ACTS, COST). The results of his research activity may be found in several presentations in national and international conferences as well as in published papers.

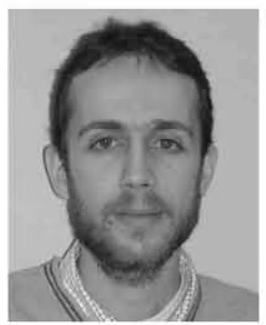

Ramón Martínez was born in Madrid, Spain, in 1975. He received the ingeniero de telecomunicación degree and the Doctor ingeniero de telecomunicación degrees in 1999 and 2004 from the Universidad Politécnica de Madrid (UPM), Spain.

In 1999, he joined the Departamento de Señales, Sistemas y Radiocomunicaciones of the E.T.S.I. de Telecomunicación School at the same University, where he currently as Associate Professor. He has published several papers in international conferences and journals, and has coauthored three books on simulation of communication systems. He has developed his professional activity in the area of satellite communications, antenna design, powerline communications (PLC) systems and and the implementation of software-defined radio prototypes. He has participated in official national and European projects (FP6, COST), and with private companies. His main research areas are smart antennas arrays for mobile communication systems and satellite communications. 